\title{
FPGA Based Linear Heating System for Measurement of Thermoluminescence
}

\author{
N. Bharathi ${ }^{1}$, P. Neelamegam ${ }^{2}$ \\ ${ }^{1}$ School of computing, SASTRA University, Thanjavur, 613401, TamilNadu, India, bharathi_n@cse.sastra.edu \\ ${ }^{2}$ School of EEE, SASTRA University, Thanjavur, 613401, TamilNadu, India, neelkeer@yahoo.com
}

A linear heating system has been developed using field programmable gate array (FPGA) for the measurement of thermoluminescence (TL) in alkali halides and other related compounds. Thermoluminescence plays an essential part in radiation dosimetry. This system measures the temperature and the amount of light emitted by the sample for TL studies. This work proposed an architectural framework, verilog HDL code for protocols for reading temperature from Thermocouple through ADC, processing of data (amount of light energy) in FPGA, sending control signal to the heater and displaying the temperature in LCD. This work shows performance improvement 3 times in comparison with conventional embedded system. The power consumption is also comparatively lower.

Keywords: Thermocouple, field programmable gate array, linear system, thermoluminescence, temperature measurement

\section{INTRODUCTION}

$\mathrm{T}$ HERMOLUMINESCENCE is a form of luminescence in which light energy is released from the substances subjected to exposure of high-energy radiation. Heating the substances to a temperature of $300^{\circ} \mathrm{C}$ emits light energy. The intensity of emission is proportional to the time duration of exposure to radiation and this is used in dating various archaeological artifacts. A thermoluminescence dosimeter, or TLD, measures ionizing radiation exposure by measuring the amount of visible light emitted from sample when it is heated linearly. Hence, it is essentially needed both for environmental monitoring and for staff personnel in facilities involving radiation exposure, among other applications. TL technique is used in many fields like geology [1], archaeology [3], [5], radiation dosimetry [2], [4] and forensic science and quality control industry.

The emergence of FPGA bridges the gap between the processing power of hardware and the flexibility of software, providing a new platform for the development of computation intensive applications. FPGAs are gaining momentum as a technology that can accelerate Moore's Law and provide greater computational capability with lower power consumption. Factors, which decide the performance of FPGA, are the overall architecture, the switch device, routing structure and logic module architecture. FPGA is utilized in this work because it has the advantages like testing, rapid prototyping and [11] its ability to transform digital design directly to Application Specific Integrated Circuits (ASICs).

This paper explains a linear heating system using FPGA for the measurement of thermoluminescence. Linear heating is necessary for TL measurements because constant increase in temperature is required. It is normally performed using a low mass heater strip made of high resistance alloys by feeding a controlled current through the heating element [12],[13]. The experimental setup is illustrated and the algorithm needed to control the temperature of the heating system linearly is elucidated. The software implementation and the results obtained are also discussed.

\section{EXPERIMENTAL SETUP}

The FPGA based system has 2 units: temperature measurement unit and Heat controlling unit. The temperature measurement unit consists of $\mathrm{K}$ type Thermocouple, photo multiplier tube (PMT), operational amplifier, MCP3202 A/D converter and Spartan 3E FPGA. The Heat controlling unit is built with the solid-state controller, which receives control signals from FPGA and controls the temperature. Fig. 1 shows the block diagram of the linear heating system.

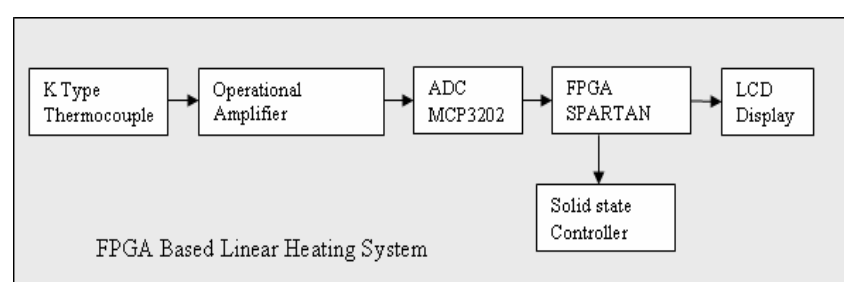

Fig.1. Block diagram of the linear heating system

\section{TEMPERATURE MEASUREMENT UNIT}

The temperature measurement unit is shown in Fig.2. Heating element (sample holder) made of kanthol metal strip of size $3 \times 1.5 \mathrm{~cm}$ is used to heat the sample. The PMT is used as a sensor to measure the amount of light emitted by the sample. The sample holder and photo multiplier tube are kept in a metal box, such that no external light can enter the box. A drawer arrangement is also made available to place the sample in the sample holder. The thermocouple is spot welded on the bottom of the sample holder (kanthol metal strip) to measure the temperature of the sample. A $2 \mathrm{~V}, 40 \mathrm{~A}$ transformer is used to heat the kanthol strip and hence the sample.

The signal generated by the junction of the thermocouple due to thermal changes is fed to an amplifier circuit. The output signal is amplified to a suitable level by using LM318 operational amplifier. Room temperature compensation is implemented using the preset arrangement 
R6. The amplified signal is sent to ADC MCP3202 to convert it into digital data. MCP3202 [6] is a successive approximation 12-bit Analog-to-Digital (A/D) Converter with on-board sample and hold circuitry. It converts the measured analog signal into digital value. Communication with the device is done using a simple [4], [6] serial interface compatible with the SPI protocol. The device is capable of sample rates of up to $50 \mathrm{ksps}$. It operates over a broad voltage range $(2.7 \mathrm{~V}-5.5 \mathrm{~V})$. The Spartan 3E FPGA issues a chip select and signal to Din pin and to read data serially through Dout pin. A semiconductor temperature sensor AD590 is used to simulate a reference junction and photo multiplier circuit to measure the light intensity, which is emitted from the sample [9] and light intensity, is read by the FPGA.

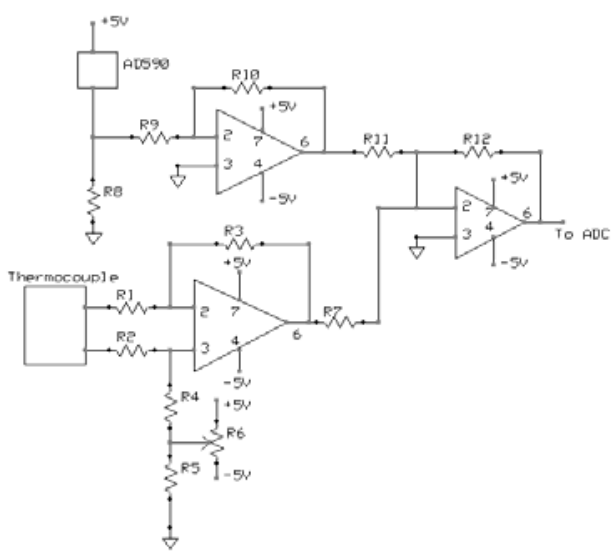

Fig.2. Temperature measurement unit

\section{HEAT-CONTROLLING UNIT}

The heat-controlling unit is used to control the power supplied to the heater. The circuit diagram for the heat control system is shown in Fig.3. The process of controlling requires a control signal from the FPGA. To issue a control signal, the FPGA needs measured temperature from the thermocouple and the actual temperature value. The actual temperature is obtained in 2 ways. One way is to store it in a look up table. A look up table is maintained in the FPGA for processing. The actual temperature data, which is needed for the linear heating system, is stored in that look up table. The other way [7] is to calculate linearly increased temperature using the temperature-time parameters:

$$
\mathrm{AT}=\mathrm{T}_{0}+\alpha \mathrm{t} .
$$

The actual temperature value is stored or calculated by increasing the temperature value with time. Depending upon the difference in measured temperature and actual temperature, the FPGA will send the control signal to the triac through optocouple MOC 3041. If the actual temperature is greater than the measured temperature, then 'on' signal is issued to the heater and if the actual temperature is lesser than the measured temperature, then 'off' signal is issued. A two-row 16 characters LCD display from Hitachi is interfaced with the FPGA through the buffer HD74HC244 to allow user communications and to display the temperature. FPGA sends the control and data signals to enable and display the data, respectively. The interfacing and conversion mechanism is implemented to display the desired characters on the LCD display.

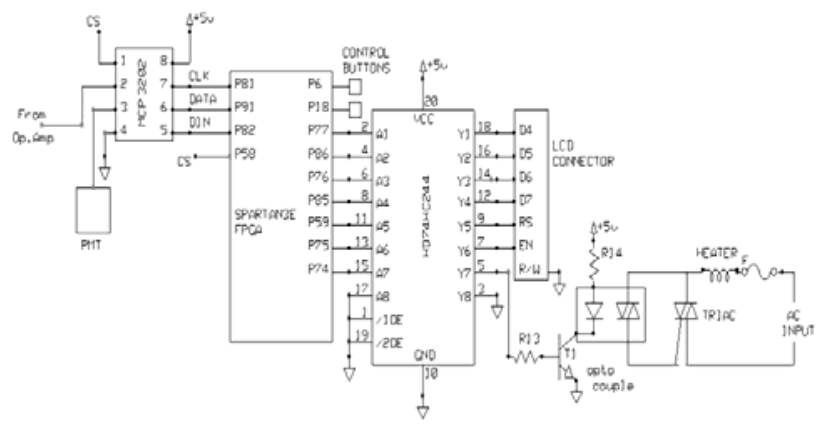

Fig.3. Circuit diagram of FPGA based linear heating system

\section{SOFTWARE IMPLEMENTATION}

Software is developed in verilog HDL, to activate MCP3202 ADC using chip select signal form the FPGA, to initialize the LCD display, to read heating rate (HR) and maximum temperature (MAXT) using control buttons, selection of temperature or light intensity, to start ADC conversion, to check end of conversion, to read data from $\mathrm{ADC}$, to measure the temperature of the sample and light emitted by the sample, to control linear heating, data computation for acquired data, storage of data for temperature and light intensity, issuing control signal from the FPGA to control the heating system, switching OFF the heater when the maximum temperature is reached. TL data are collected each time the LCD display is updated. Temperature against time and TL against temperature points are stored at the same time. The free ISE/WebPack CAD software from Xilinx is used for simulation and also to create bit files from verilog source code. Digilent's PCbased program called Adept is used to configure the FPGA with any suitable bit file stored on the computer. Adept uses a USB cable to transfer a selected bit file from the PC to the FPGA board.

\section{Algorithm:}

Step 1: Initialize LCD display.

Step 2: Read the maximum temperature (MAXT) and the heating rate (HR).

Step 3: Load the calibration table for linear temperature.

Step 4: Send start signal to ADC.

Step 5: Select thermocouple channel ( $\mathrm{CH} 0)$.

Step 6: Read the sample temperature (MT).

Step 7: Output temperature (MT) in LCD.

Step 8: Call Decision (MT, AT, MAXT) for linearity.

Step 9: Send the control signal to the heater.

Step 10: Select PMT channel $(\mathrm{CH} 1)$ and measure light intensity.

Step 11: Wait for time delay.

Step 12: Go to step 4.

Decision (MT, AT, MAXT)

Step 1: If (MT > = AT) then return 'off' signal.

Step 2: If $(\mathrm{MT}<\mathrm{AT})$ then return 'on' signal.

Step 3: If $(\mathrm{MAXT}<\mathrm{MT})$ stop. 


\section{RESULTS AND DISCUSSION}

The linear heating can be varied from 10 to $120 \mathrm{deg} / \mathrm{min}$. Fig. 4 shows the graph with the increase in temperature with respect to time, for both FPGA and non-FPGA based systems. The non-FPGA based system used 8031microcontroller board with its peripherals. The measurement system was configured to operate over the temperature range of 20 to $300^{\circ} \mathrm{C}$. The linear heating of the system was tested and is shown in Fig.5. The error in temperature measurement was found to be within $0.1 \%$ in the FPGA based system. The performance of the FPGA based instrument has been evaluated using gamma irradiated undoped $\mathrm{CsBr}$ samples and readings obtained agree with $\mathrm{S}$. Selvasekarapandian et.al [7],[8]. The use of FGPA reduces the power consumption and the processing speed is increased about 3 times.

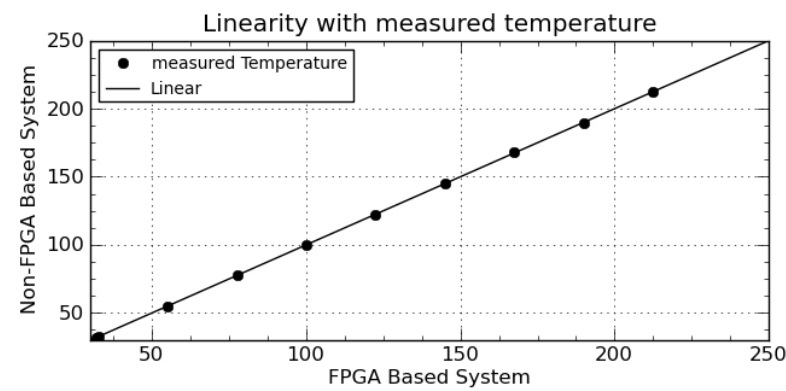

Fig.4. measured temperature for FPGA and non-FPGA based system

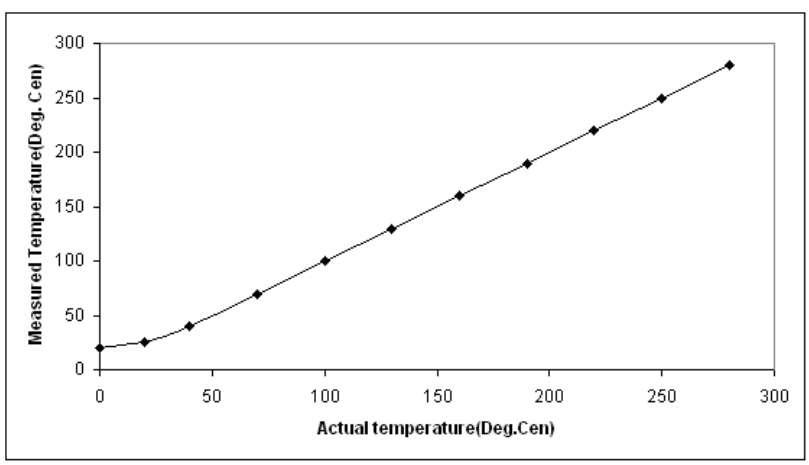

Fig.5. Linear heating of the system

\section{CONCLUSION}

The FPGA based linear heating system results show good performance for thermoluminescence measurements. The use of a FPGA, the process of temperature control, data acquisition and data storage will automate data computation and presentation. Moreover, the system is easily operated and does not require any programming expertise. The instrument was also tested with different samples to check the reproducibility. For evaluation of linearity, the results are compared with a conventional system for linear heating, the measured data are used for the regression analysis and it reveals excellent correlation. The correlation coefficient (r) is found to be 0.9998 . It also proves the reliability of the system for linear heating and radiation dosimetry as well as its suitability for a wide range of applications. In comparison to conventional systems, the system is well capable and portable for TL measurements.

\section{REFERENCES}

[1] Deckers, K., Sanderson, D.C.W., Spencer, J.Q. (2005). Thermoluminescence screening of non-diagnostic sherds from stream sediments to obtain a preliminary alluvial chronology: An example from Cyprus. Geoarchaeology, 20, 67-77.

[2] Botter-Jensen, L., McKeever, S.W.S., Wintle, A.G. (2003). Optically Stimulated Luminescence Dosimetry. Amsterdam: Elsevier.

[3] Richter, D., Krbetschek, M. (2006). A new thermoluminescence dating method for heated flint. Archaeometry, 48, 695-705.

[4] Richter, D., Temming, H. (2006). Dose recovery tests for heated flint. Testing equivalent dose protocols. Radiation Measurements, 41, 819-825.

[5] Richter, D. (2007). Advantages and limitations of thermoluminescence dating of heated flint from Palaeolithic sites. Geoarchaeology, 22, 671-683.

[6] Microchip Technology Inc. (2006). MCP3202. Device Document. http://ww1.microchip.com/downloads/en/ DeviceDoc/ 21034D.pdf

[7] Zhan, X., Yin, G., Ma, B. (1995). New heating controller and computation for linear heating stability experiment. International Journal of Pharmaceutics, $115,161-166$.

[8] Selvasekarapandian, S., Christober Selvan, P., Neelamegam, P. (1996). Thermoluminescence studies on $\mathrm{Sr} 2+$ doped cesium halides and their application to dosimetry. Nuclear Instruments and Methods in Physics Research, 111 (3-4), 290-296.

[9] Selvasekarapandian, S., Neelamegan, P., Christober Selvan, P. (1992). Thermoluminescence of gammairradiated $\mathrm{CsBr}: \mathrm{Ba} 2+$ and $\mathrm{CsCl}: \mathrm{Ba} 2+$ crystals and its application to dosimetry. Journal of Luminescence, 54 (2), 121-129.

[10] Chandok, R.S., Kaur, R., Chandok, G.K., Chandra, B.P. (2006). Temperature effect on lyoluminescence of potassium halide microcrystals in luminol solution. Indian Journal of Pure \& Applied Physics, 44, 519-523.

[11] Stojanović, R., Karadaglić, D., Mirković, M., Milošević, D. (2011). A FPGA system for QRS complex detection based on Integer Wavelet Transform. Measurement Science Review, 11 (4), 131-138.

[12] Bøtter-Jensen, L., McKeever, S.W.S., Wintle, A.G. (2003). Optically Stimulated Luminescence Dosimetry. Elsevier.

[13] Chen, R., McKeever, S.W.S. (1997). Theory of Thermoluminescence and Related Phenomena. World Scientific Publishers.

Received August 17, 2011. Accepted December 12, 2011. 